OPEN ACCESS

Edited by:

Yunlong Yang,

Fudan University, China

Reviewed by:

Laura Bowers,

Purdue University, United States

Hamid Morjani,

Université de Reims

Champagne-Ardenne, France

*Correspondence:

Si Sun

59333173@qq.com

Shengrong Sun

sun137@sina.com

${ }^{t}$ These authors have contributed equally to this work

Specialty section:

This article was submitted to Molecular and Cellular Oncology, a section of the journal Frontiers in Cell and Developmental Biology

Received: 16 February 2020 Accepted: 02 April 2020

Published: 28 April 2020

Citation:

Wu Q, Li B, Sun S and Sun S (2020) Unraveling Adipocytes and Cancer Links: Is There a Role for Senescence?

Front. Cell Dev. Biol. 8:282. doi: 10.3389/fcell.2020.00282

\section{Unraveling Adipocytes and Cancer Links: Is There a Role for Senescence?}

\author{
Qi Wu ${ }^{1,2 t}$, Bei Li" ${ }^{1+}$, Si Sun ${ }^{3 *}$ and Shengrong Sun ${ }^{1 *}$ \\ 1 Department of Breast and Thyroid Surgery, Renmin Hospital of Wuhan University, Wuhan, China, ${ }^{2}$ Faculty of Medicine, \\ University of Paris Sud-Saclay, Le Kremlin-Bicêtre, France, ${ }^{3}$ Department of Clinical Laboratory, Renmin Hospital of Wuhan \\ University, Wuhan, China
}

Senescence is characterized by a permanent cell cycle arrest that is elicited in response to different stresses. In addition, senescent cells undergo multiple other phenotypic alterations, such as autophagy modulation, metabolic reprogramming, and the senescence-associated secretory phenotype (SASP). These senescence-related and inflammatory effects prevail within tumors and are strongly controlled by cancer properties, and inflammatory mediators further maintain and propagate the senescence process to adjacent cells. It is important to consider these detrimental effects that may drive tumorigenesis or cancer relapse. Importantly, cancer-associated adipocytes (CAAs) are one of the primary stromal cells in various tumor microenvironments and favor tumor progression by releasing various factors that can mediate local and systemic effects. However, it remains unclear whether CAAs possess senescent features. In this review, we discuss the complex relationship between senescence and CAAs and highlight important considerations for therapeutics.

Keywords: adipocytes, cancer, senescence, tumor microenvironment (TEM), inflammation

\section{INTRODUCTION}

Adipocytes are one of the primary stromal cells present in multiple cancers, such as breast, ovarian and colorectal cancer (Nieman et al., 2011; Wu et al., 2019a). Specifically, cancer-associated adipocytes (CAAs) are considered to play an active role in the tumor microenvironment. CAAs are capable of exhibiting several phenotypes, which, in aggregate, define their state. Morphologically, CAAs have a decreased cell size and a dilated interstitial space and exhibit a dramatic loss of lipid content (Lapeire et al., 2014). In terms of specific markers, CAAs show a reduction in adipose markers such as hormone-sensitive lipase, resistin, and adipocyte fatty acid-binding protein but a remarkable increase in fibroblast-like biomarkers such as fibroblast activation protein $\alpha$, chondroitin sulfate proteoglycan, and $\alpha$ smooth muscle actin (Jung et al., 2015). Compared to other cells CAAs secrete more chemokine (C-C motif) ligand 2 (CCL2), chemokine (C-C motif) ligand 5 (CCL5), interleukin-6 (IL-6), tumor necrosis factor-alpha, vascular endothelial growth factor (VEGF), and leptin but less adiponectin (Dirat et al., 2011; Wu et al., 2019a). In terms of metabolic changes, CAAs enhance various catabolic processes and release high-energy metabolites, such as lactate, pyruvate, free fatty acids, and ketone bodies (Wu et al., 2019a). Interestingly, skeletal muscle cells are induced to undergo apoptosis via tumor-derived microvesicles (He et al., 2014), while the adipocytes surrounding the tumor cells present smaller sizes with a dilated interstitial space 
(Dirat et al., 2011). Hence, CAAs possess substantial remodeling capacity but can still survive while under attack from tumor cells. The potential conversion of normal adipocytes to CAAs deserves further exploration.

Cellular senescence was originally identified as stable growth arrest, and it is further thought to be a stress response that can be triggered by a variety of intrinsic and extrinsic types of damage, such as oncogenic activation, oxidative and genotoxic stress, mitochondrial dysfunction, irradiation, and chemotherapeutic agents (Herranz and Gil, 2018). Many phenotypic alterations that are relevant to senescent processes are associated with the pathophysiological effects of senescent cells. For instance, senescent cells undergo a series of alterations, such as morphological changes, chromatin remodeling, and metabolic reprogramming, culminating in the senescenceassociated secretory phenotype (SASP), which is described as the complex secretion of mostly proinflammatory factors (Rodier and Campisi, 2011). In addition, senescent cells in the surrounding microenvironment have tumor-promoting effects. In coculture experiments, senescent fibroblasts have been shown to regulate the growth of prostate epithelial cells with mutations, inducing the generation of preneoplastic cells (Bavik et al., 2006). Interestingly, senescent cells within the microenvironment and their functionally analogous cousins, cancer-associated fibroblasts (CAFs), promote transformation by stimulating tumor growth, angiogenesis, and invasion (Ruhland et al., 2016; Milanovic et al., 2018). The broad actions of CAFs and senescent cells in tumor progression have been ascribed to the plethora of tumorigenic factors that these cell types secrete (Alspach et al., 2013). However, whether CAAs in the tumor microenvironment undergo senescent transformation or possess some senescent characteristics remains unclear.

Adipose tissue plays a central role in longevity and age-related disorders (Tchkonia et al., 2010). The dysfunction of senescent fat tissues contributes to age-related chronic inflammation, diabetes, and cancer (Martyniak and Masternak, 2017; Sieben et al., 2018; Ghosh et al., 2019). Recent evidence has indicated that fat cells can be switched into a proinflammatory, dysfunctional, senescent-like state in individuals with obesity (Tchkonia et al., 2010; Ghosh et al., 2019). However, this would result in a significant reduction in the amount of proinflammatory cytokines (CCL2 and IL-6) derived from the visceral adipose tissue (VAT) of aging mice and decreases in the levels of cyclindependent kinases (p16 and p21) cultured in fetal-derived serum (Ghosh et al., 2019). This suggests that fetal plasma rescues the senescent phenotypes of VAT in old mice. Additionally, cellular senescence is regarded as an adaptive response to stress during which multiple cellular functions, including metabolic processes and secretome activity, have been readjusted (Herranz and Gil, 2018). Furthermore, autophagy and endoplasmic reticulum (ER) stress are two of the major cellular stress responses (Galluzzi et al., 2018). It is evident that increased expression of ER stress markers such as GRP78 and CHOP is observed in the adipose tissue of old mice, and activation of the ER stress response contributes to the elevated secretion in CCL2 and IL-6 (Ghosh et al., 2015). Likewise, autophagic function is diminished in the adipose tissue of aging mice, and impaired autophagy further facilitates ER stress (Ghosh et al., 2016). Taken together, these data indicate that disorganized stress responses destroy adipose tissue homeostasis and exacerbate age-associated inflammation. Here, we speculate the feasible molecular mechanisms that connect CAAs with cellular senescence.

\section{AUTOPHAGY}

Autophagy is a mechanism for isolating and degrading various cytoplasmic structures, such as damaged organelles and invasive microorganisms, via lysosomes. Activated autophagy in the stroma promotes cancer progression. For example, high expression of Beclin-1, an inducer of autophagy, promotes the growth of fibroblasts in breast cancer (Capparelli et al., 2012). For human fibroblasts, activated autophagy results in their senescence (Young et al., 2009); meanwhile, CAFs from multiple cancers are found to exhibit senescent phenotypes (Dou et al., 2017). This evidence demonstrates that autophagy may play a crucial role in the malignant transformation and senescence of stromal cells. Moreover, there are several autophagyassociated signals in the stroma connecting senescence and the malignant transformation of adipocytes. First, caveolin-1 (Cav-1), a main structural protein of caveolae, plays an important role in membrane transport (endocytosis and transcytosis), the maintenance of membrane lipid composition and signal transduction within cells (Wang et al., 2017). Intriguingly, Cav-1 is widely considered a tumor suppressor, and low expression of Cav-1 in surrounding adipocytes drives tumor growth and metastasis in breast cancer (Witkiewicz et al., 2009; Wu et al., 2019b). Under hypoxic conditions, Cav-1 can be degraded in the autophagic response via activation of hypoxia inducible factor- $1 \alpha$ (HIF-1 $\alpha$ ) and NF-KB (Martinez-Outschoorn et al., 2010). Recently, Cav-1 downregulation has been shown to contribute to the induction of cellular senescence in fibroblasts; Cav-1 deficiency impaired mitochondrial respiration and inactivated silent information regulator 2 homolog 1 (SIRT1) to promote premature senescence (Yu et al., 2017). Therefore, Cav-1-induced autophagy may be the crucial link between CAAs and cellular senescence. In addition, transforming growth factor $\beta$ (TGF$\beta$ ) is a multifunctional cytokine that not only modulates the growth and differentiation of cancer cells but also mediates the senescence of cancer stroma and autophagy (Kiyono et al., 2009; Hassona et al., 2013). Furthermore, TGF- $\beta$ activates autophagy by inducing the accumulation of autophagosomes and converting microtubule-associated protein 1 light chain 3 to its lipid-modified form to promote the degradation of proteins (Kiyono et al., 2009). In addition, TGF- $\beta$ accelerates fibroblast senescence by increasing reactive oxygen species (ROS) levels to reinforce malignant behavior (Hassona et al., 2013). TGF- $\beta$ overexpression in conditioned media from breast cancer cells promoted a reversal of adipose cells to a fibroblastic phenotype (Guerrero et al., 2010). These results reveal the functional diversity of CAAs, suggesting that CAAs may enhance malignant behavior by inducing the senescence of adipocytes via TGF- $\beta$-dependent autophagy. Ultimately, the link between CAAs and senescence has been demonstrated in adipocytes with 
overexpression of HIF- $1 \alpha$, which facilitates autophagy and tumor growth (Diedrich et al., 2016; Wu et al., 2019b). Likewise, von Hippel-Lindau (VHL), a tumor suppressor gene, is a major regulator of HIF- $1 \alpha$ subunits under aerobic conditions, and it directs proteasome-mediated degradation to inactivate HIF-1 $\alpha$ function. It has been found that the absence of VHL induces senescence via regulation of $\mathrm{p} 400$ and $\mathrm{p} 27$, both of which enhance the ability of pRB to promote cell cycle arrest and senescence; this activity occurs in a HIF- $\alpha$-dependent manner (Young et al., 2008).

In summary, studying autophagy in CAAs enables us to recognize the link between autophagy and senescence among these different compartments (Figure 1). In certain cancer subtypes, the activation of autophagic flux in the stroma is an indicator of senescence as well as a tumor promoter. Drugs that target autophagy or senescence are effective antitumor agents, and determining the autophagy or senescence status in the stroma may function as a potential biomarker.

\section{SASP}

The SASP suggests that senescent cells communicate with neighboring cells to spread the stress response and influence their microenvironment. The SASP increases the expression of a range of chemokines (chemoattractants and macrophage inflammatory proteins), proinflammatory cytokines (most notably IL-1 $\beta$, IL-6, and IL-8), growth factors (TGF- $\beta$, granulocyte-macrophage colony-stimulating factor, and hepatocyte growth factor), and matrix-remodeling enzymes (Lasry and Ben-Neriah, 2015; Herranz and Gil, 2018). Interestingly, recent work has shown that prior to the arrival of metastatic cells at a distal site, numerous changes that prepare the site for the growth of the tumor cells occur. Outstanding questions in this process include how the microenvironment changes at these distal sites and what cell types are responsible for these changes. Because senescent cells accumulate in tissue with age (Krtolica et al., 2001), these observations raise the possibility that senescent cells within the microenvironment may have the potential to condition the pre-metastatic niche and increase the likelihood of the establishment of metastases. Moreover, attention has been paid to investigating the underlying mechanisms that induce the appearance of cancer-initiating cells or cancer stem cells (CSCs), which seem to drive the formation of distant metastasis (Batlle and Clevers, 2017). Intriguingly, chemokines secreted by senescent cells have been shown to select for CSCs (Milanovic et al., 2018). As one of the most strongly expressed SASP factors, IL-6 has crucial effects in regulating the self-renewal of breast CSCs (Korkaya et al., 2011). Similarly, analyses of breast CAFs recently revealed CCL2 expression can stimulate CSC-like properties, including increased sphereforming capacity and self-renewal activity (Tsuyada et al., 2012; Milanovic et al., 2018). The interleukins IL-6 and IL-8 facilitate the growth of breast cancer epithelial cells. Treatment with neutralizing antibodies against IL-6 and IL-8 decreased the promotion of growth of preneoplastic epithelial cells cocultured with senescent cells (Coppe et al., 2008). Furthermore, treatment with recombinant IL-6 and IL-8 could effectively promote the growth of breast cancer epithelial cells (Ortiz-Montero et al., 2017). Moreover, senescent cells could facilitate epithelial-tomesenchymal transition (EMT), which plays an important role in tumor cell metastasis. Previous work has demonstrated that after treatment with conditioned media from senescent fibroblasts, human breast cancer cell lines showed decreased expression of cytokeratin and E-cadherin, which are hallmarks of EMT (Coppe et al., 2008). Senescent cells induce the upregulation of EMT via matrix metalloproteinase 3 (Parrinello et al., 2005). In addition, senescent fibroblasts promote the invasion of human umbilical vascular endothelial cells (HUVECs) in vitro and facilitate the vascularization of tumors in xenograft experiments by secreting VEGF (Coppe et al., 2006). Ultimately, sFRP2, as a Wnt antagonist, can be secreted by aging fibroblasts. sFRP2 promotes the loss of APE1, a key redox effector, in melanoma cells by decreasing the levels of $\beta$-catenin and microphthalmiaassociated transcription factor (MITF). Furthermore, the increase in sFRP2 endows melanoma cells with the capacity to resist targeted therapy and metastasis (Kaur et al., 2016). In conclusion, these results reveal that senescent cells promote the establishment of primary tumors by expressing SASP factors.

Similar to senescent fibroblasts within the tumor microenvironment, CAAs stimulate tumor growth, angiogenesis, invasion, and metastasis to facilitate the transformation process (Dirat et al., 2011; Lapeire et al., 2014; Wu et al., 2019a). The tumor-promoting activity of CAAs is partially mediated by an altered secretion phenotype that overlaps strongly with the SASP (Figure 1). Indeed, several studies have demonstrated that the expression profile of CAAs is enriched in many of the same proinflammatory factors, such as IL-6, IL-8, and a variety of CCLs, that are present in the SASP (Dirat et al., 2011; Vazquez Rodriguez et al., 2018; Wu et al., 2019b). Thus, it was not surprising that similar to senescent cells, CAAs also express increased levels of MMPs (Dirat et al., 2011; Rowan et al., 2014), which can enhance EMT to promote tumor metastasis. In addition, adipose-derived stem cells treated with tumor-derived extracellular vesicles could secrete more VEGF to promote angiogenic sprouting of HUVECs (Song et al., 2017). Given the phenotypic similarities and emerging molecular similarities between senescent cells and CAAs, we have argued that CAAs may be an operational subtype of senescent cells.

\section{ONCOGENE-INDUCED SENESCENCE}

Tumor-transformed adipocytes have been demonstrated to form via the expression of various tumor-derived inflammatory factors and contain multiple exosomes (Petruzzelli et al., 2014; Wu et al., 2018, 2019c). Likewise, in response to the activation of many oncogenes, normal cells must undergo cellular senescence. For example, when an oncogenic form of RAS was expressed in human fibroblasts, oncogene-induced senescence (OIS) was originally observed (Serrano et al., 1997). The number of oncogenes able to induce senescence has risen to $\sim 50$ oncogenes (Gorgoulis and Halazonetis, 2010). 


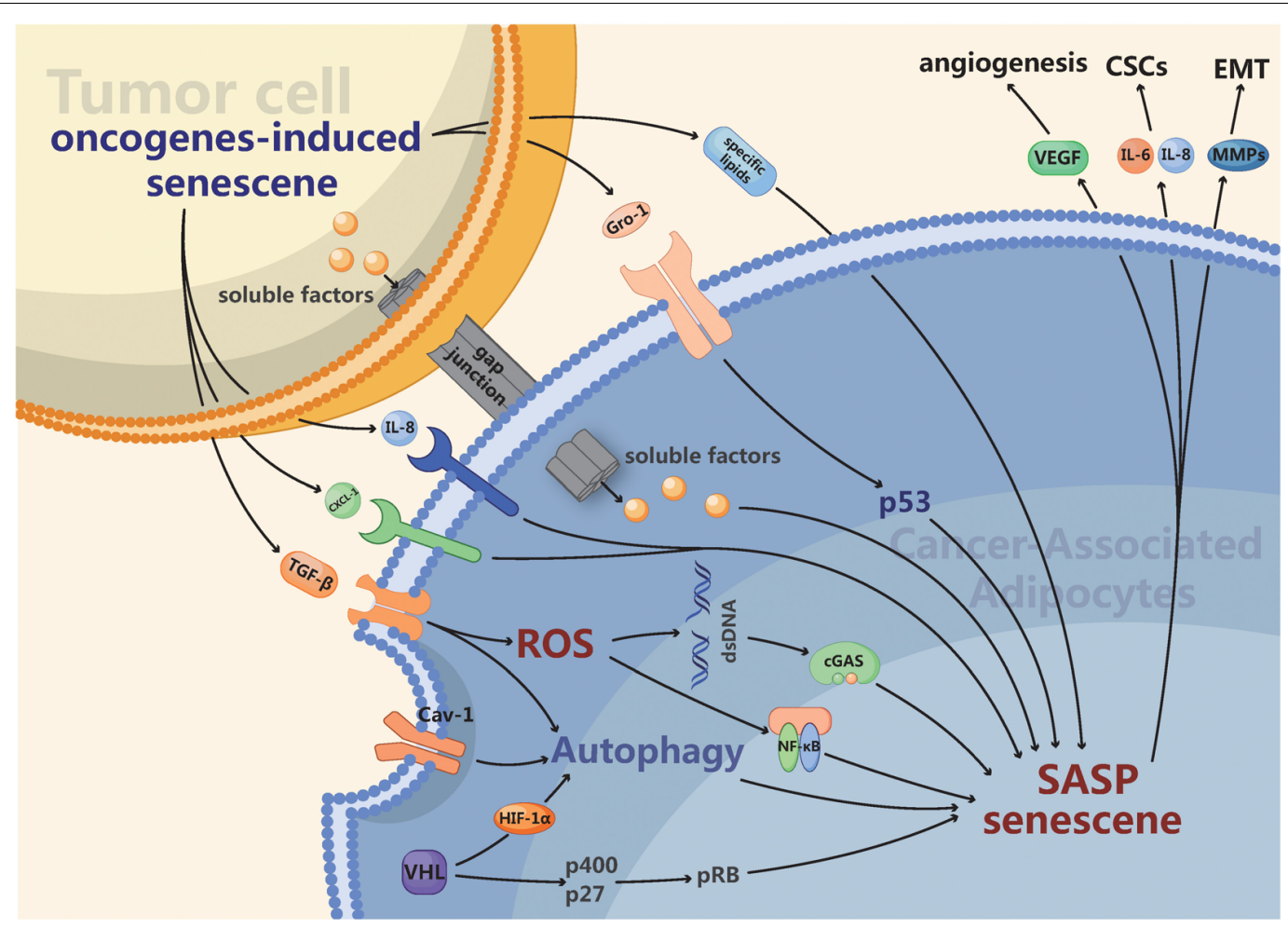

FIGURE 1 | The intrinsic and extrinsic mechanisms connecting cancer-associated adipocytes (CAAs) with senescence. Firstly, Autophagy activated by diverse intracellular and extracellular stress like hypoxia in CAAs is a hallmark of senescence as well as a tumor promoter. Furthermore, the secreted profile of CAAs is enriched in many of the same pro-inflammatory factors, such as IL-6, IL-8, and a variety of chemokines, which overlap strongly with the senescence-associated secretory phenotype (SASP). Finally, the activation of several oncogenes such as RAS or the loss of tumor suppressors like p53 can promote senescence. The senescent CAAs may arise from gap junctions and paracrine signals originating from tumor cells or other senescent cells. Ultimately, CAAs possessing multifarious senescent properties may enhance tumor angiogenesis, proliferation of cancer stem cells (CSCs) and epithelial-mesenchymal transition (EMT).

In addition, the loss of tumor suppressors can promote senescence, such as the loss of PTEN (Alimonti et al., 2010), P53 (Acosta et al., 2013), or VHL (Young et al., 2008). Cellular senescence can also be induced by established tumors or neighboring neoplastic cells, as demonstrated by a study in which senescence was induced in the stroma surrounding tumors through injection of tumor cells (Burd et al., 2013). Mechanistically, OIS can induce senescence of neighboring cells through induction of SASP or gap junction-mediated cell-cell contact, thereby enhancing its own effects (Nelson et al., 2012; Acosta et al., 2013). Indeed, several studies have demonstrated that numerous SASP factors, including TGF- $\beta$ (Acosta et al., 2013), IL-8, and CXCL1 (Acosta et al., 2008), and several SASP pathways, such as NF- $\kappa$ B signaling activated by ROS (Nelson et al., 2018) and cGAS-STING signaling (Yang et al., 2017), can mediate the induction of paracrine senescence. Cancer cells produce ROS and increase oxidative stress in adjacent adipocytes (Ladanyi et al., 2018), and ROS are necessary and sufficient to activate NF- $\mathrm{B}$, which promotes SASP and results in the DNA damage response in CAAs (Nelson et al., 2018). Moreover, with the accumulation of DNA products from impaired synthesis in the cytoplasm, cGAS, as a cytosolic DNA sensor, is essential for senescence (Yang et al., 2017). In addition, chemokine growth-regulated oncogene 1 (Gro-1) was activated by RAS and upregulated in serum samples from ovarian cancer patients. Furthermore, Gro-1 could facilitate the senescence of stromal fibroblasts by affecting functional p53 to promote tumor growth (Yang et al., 2006). Extracellular vesicles also play an important role in paracrine signaling by transferring contents to impact recipient cell signaling. A recent study indicated that extracellular vesicles derived from cells with H-Rasinduced senescence were enriched and capable of transporting multiple specific lipids, including hydroxylated sphingomyelin, sphingomyelin, lysophosphatidic acid, and sulfatides (Buratta et al., 2017). In summary, the paracrine senescence induced by OIS reveals a mechanism by which senescent adipocytes can gain enhanced effects and may amplify negative effects on tumors (Figure 1).

\section{CONCLUDING REMARKS AND FUTURE DIRECTIONS}

Cellular senescence, a typical characteristic of cancer, can be triggered by a variety of mechanisms in and around tumors and has deleterious effects on tumor initiation, growth, recurrence, and therapeutic efficacy. However, the senescent characteristics 
of adipocytes and the mechanisms by which they affect the phenotype of tumor cells remain incompletely understood. Indeed, autophagy, a central regulator in senescent cells, mediates the functions of CAAs. Therefore, further characterization of autophagy in CAAs and cancer is crucial for us to understand these mechanisms and develop targeted therapeutic approaches in the future. Additionally, it is important to again note the similarities between the SASP and the expression profile of CAAs. The overlap in expression profiles between these two tumor-promoting cell types suggests that the regulatory pathways that are further characterized in senescent cells will be directly applicable in CAAs. Given the potent tumor-promoting nature of the SASP, identifying the regulatory mechanisms that govern the expression of genes related to this phenotype will contribute to the development of stroma-targeting cancer therapies. This concept is consistent with our speculation that a subset of senescent CAAs is present and that OIS may be a major contributor to the generation of senescent adipocytes. The current challenge in the field is to discern which changes are critical for tumor progression and to determine how cellautonomous mutations within incipient tumor cells influence stromal changes.

\section{REFERENCES}

Acosta, J. C., Banito, A., Wuestefeld, T., Georgilis, A., Janich, P., Morton, J. P., et al. (2013). A complex secretory program orchestrated by the inflammasome controls paracrine senescence. Nat. Cell Biol. 15, 978-990. doi: 10.1038/ ncb2784

Acosta, J. C., O’Loghlen, A., Banito, A., Guijarro, M. V., Augert, A., Raguz, S., et al. (2008). Chemokine signaling via the CXCR2 receptor reinforces senescence. Cell 133, 1006-1018. doi: 10.1016/j.cell.2008.03.038

Alimonti, A., Nardella, C., Chen, Z., Clohessy, J. G., Carracedo, A., Trotman, L. C., et al. (2010). A novel type of cellular senescence that can be enhanced in mouse models and human tumor xenografts to suppress prostate tumorigenesis. J. Clin. Investig. 120, 681-693. doi: 10.1172/JCI4 0535

Alspach, E., Fu, Y., and Stewart, S. A. (2013). Senescence and the pro-tumorigenic stroma. Crit. Rev. Oncog. 18, 549-558.

Batlle, E., and Clevers, H. (2017). Cancer stem cells revisited. Nat. Med. 23, 1124-1134. doi: 10.1038/nm.4409

Bavik, C., Coleman, I., Dean, J. P., Knudsen, B., Plymate, S., and Nelson, P. S. (2006). The gene expression program of prostate fibroblast senescence modulates neoplastic epithelial cell proliferation through paracrine mechanisms. Cancer Res. 66, 794-802.

Buratta, S., Urbanelli, L., Sagini, K., Giovagnoli, S., Caponi, S., Fioretto, D., et al. (2017). Extracellular vesicles released by fibroblasts undergoing H-Ras induced senescence show changes in lipid profile. PLoS One 12:e0188840. doi: 10.1371/ journal.pone. 0188840

Burd, C. E., Sorrentino, J. A., Clark, K. S., Darr, D. B., Krishnamurthy, J., Deal, A. M., et al. (2013). Monitoring tumorigenesis and senescence in vivo with a p16(INK4a)-luciferase model. Cell 152, 340-351. doi: 10.1016/j.cell.2012. 12.010

Capparelli, C., Guido, C., Whitaker-Menezes, D., Bonuccelli, G., Balliet, R., Pestell, T. G., et al. (2012). Autophagy and senescence in cancerassociated fibroblasts metabolically supports tumor growth and metastasis via glycolysis and ketone production. Cell Cycle 11, 2285-2302. doi: 10.4161/cc. 20718

Coppe, J. P., Kauser, K., Campisi, J., and Beausejour, C. M. (2006). Secretion of vascular endothelial growth factor by primary human fibroblasts at senescence. J. Biol. Chem. 281, 29568-29574.

\section{AUTHOR CONTRIBUTIONS}

QW and BL were responsible for collecting and collating documents. QW and BL were responsible for writing this review, while SiS was responsible for the revision and ShS was responsible for editing and submission. All authors read and approved the final manuscript.

\section{FUNDING}

This work was supported by a National Natural Science Foundation of China (NSFC) grant (Grant No: 81471781) to ShS. This work was also supported by a Hubei Province health and family planning scientific research project (Grant No. WJ2019Q044) and a National Natural Science Foundation of China grant (Grant No: 81903166) to SiS.

\section{ACKNOWLEDGMENTS}

We thank the professional English editor (American Journal Experts) for assistance in improving the quality of the language.

Coppe, J. P., Patil, C. K., Rodier, F., Sun, Y., Munoz, D. P., Goldstein, J., et al. (2008). Senescence-associated secretory phenotypes reveal cellnonautonomous functions of oncogenic RAS and the p53 tumor suppressor. PLoS Biol. 6:e301. doi: 10.1371/journal.pbio.0060301

Diedrich, J. D., Rajagurubandara, E., Herroon, M. K., Mahapatra, G., Huttemann, M., and Podgorski, I. (2016). Bone marrow adipocytes promote the Warburg phenotype in metastatic prostate tumors via HIF-1alpha activation. Oncotarget 7, 64854-64877. doi: 10.18632/oncotarget.11712

Dirat, B., Bochet, L., Dabek, M., Daviaud, D., Dauvillier, S., Majed, B., et al. (2011). Cancer-associated adipocytes exhibit an activated phenotype and contribute to breast cancer invasion. Cancer Res. 71, 2455-2465. doi: 10.1158/0008-5472. CAN-10-3323

Dou, Z., Ghosh, K., Vizioli, M. G., Zhu, J., Sen, P., Wangensteen, K. J., et al. (2017). Cytoplasmic chromatin triggers inflammation in senescence and cancer. Nature 550, 402-406. doi: 10.1038/nature24050

Galluzzi, L., Yamazaki, T., and Kroemer, G. (2018). Linking cellular stress responses to systemic homeostasis. Nat. Rev. Mol. Cell Biol. 19, 731-745. doi: 10.1038/ s41580-018-0068-0

Ghosh, A. K., Garg, S. K., Mau, T., O’Brien, M., Liu, J., and Yung, R. (2015). Elevated endoplasmic reticulum stress response contributes to adipose tissue inflammation in aging. J. Gerontol. Ser. A Biol. Sci. Med. Sci. 70, 1320-1329.

Ghosh, A. K., Mau, T., O’Brien, M., Garg, S., and Yung, R. (2016). Impaired autophagy activity is linked to elevated ER-stress and inflammation in aging adipose tissue. Aging 8, 2525-2537. doi: 10.18632/aging.101083

Ghosh, A. K., O’Brien, M., Mau, T., Qi, N., and Yung, R. (2019). Adipose tissue senescence and inflammation in aging is reversed by the young milieu. J. Gerontol. Ser. A Biol. Sci. Med. Sci. 74, 1709-1715. doi: 10.1093/gerona/gly290

Gorgoulis, V. G., and Halazonetis, T. D. (2010). Oncogene-induced senescence: the bright and dark side of the response. Curr. Opin. Cell Biol. 22, 816-827. doi: 10.1016/j.ceb.2010.07.013

Guerrero, J., Tobar, N., Caceres, M., Espinoza, L., Escobar, P., Dotor, J., et al. (2010). Soluble factors derived from tumor mammary cell lines induce a stromal mammary adipose reversion in human and mice adipose cells. Possible role of TGF-betal and TNF-alpha. Breast Cancer Res. Treat. 119, 497-508. doi: 10.1007/s10549-009-0491-1

Hassona, Y., Cirillo, N., Lim, K. P., Herman, A., Mellone, M., Thomas, G. J., et al. (2013). Progression of genotype-specific oral cancer leads to senescence of cancer-associated fibroblasts and is mediated by oxidative 
stress and TGF-beta. Carcinogenesis 34, 1286-1295. doi: 10.1093/carcin/bg t035

He, W. A., Calore, F., Londhe, P., Canella, A., Guttridge, D. C., and Croce, C. M. (2014). Microvesicles containing miRNAs promote muscle cell death in cancer cachexia via TLR7. Proc. Natl. Acad. Sci. U.S.A. 111, 4525-4529. doi: 10.1073/ pnas. 1402714111

Herranz, N., and Gil, J. (2018). Mechanisms and functions of cellular senescence. J. Clin. Investig. 128, 1238-1246. doi: 10.1172/JCI95148

Jung, Y. Y., Lee, Y. K., and Koo, J. S. (2015). Expression of cancer-associated fibroblast-related proteins in adipose stroma of breast cancer. Tumour Biol. 36, 8685-8695. doi: 10.1007/s13277-015-3594-9

Kaur, A., Webster, M. R., Marchbank, K., Behera, R., Ndoye, A., Kugel, C. H. III, et al. (2016). sFRP2 in the aged microenvironment drives melanoma metastasis and therapy resistance. Nature 532, 250-254. doi: 10.1038/nature 17392

Kiyono, K., Suzuki, H. I., Matsuyama, H., Morishita, Y., Komuro, A., Kano, M. R., et al. (2009). Autophagy is activated by TGF-beta and potentiates TGF-beta-mediated growth inhibition in human hepatocellular carcinoma cells. Cancer Res. 69, 8844-8852. doi: 10.1158/0008-5472.CAN-084401

Korkaya, H., Liu, S., and Wicha, M. S. (2011). Breast cancer stem cells, cytokine networks, and the tumor microenvironment. J. Clin. Investig. 121, 3804-3809. doi: 10.1172/JCI57099

Krtolica, A., Parrinello, S., Lockett, S., Desprez, P. Y., and Campisi, J. (2001). Senescent fibroblasts promote epithelial cell growth and tumorigenesis: a link between cancer and aging. Proc. Natl. Acad. Sci. U.S.A. 98, 12072-12077.

Ladanyi, A., Mukherjee, A., Kenny, H. A., Johnson, A., Mitra, A. K., Sundaresan, S., et al. (2018). Adipocyte-induced CD36 expression drives ovarian cancer progression and metastasis. Oncogene 37, 2285-2301. doi: 10.1038/s41388-0170093-z

Lapeire, L., Hendrix, A., Lambein, K., Van Bockstal, M., Braems, G., Van Den Broecke, R., et al. (2014). Cancer-associated adipose tissue promotes breast cancer progression by paracrine oncostatin M and Jak/STAT3 signaling. Cancer Res. 74, 6806-6819. doi: 10.1158/0008-5472.CAN-14-0160

Lasry, A., and Ben-Neriah, Y. (2015). Senescence-associated inflammatory responses: aging and cancer perspectives. Trends Immunol. 36, 217-228. doi: 10.1016/j.it.2015.02.009

Martinez-Outschoorn, U. E., Trimmer, C., Lin, Z., Whitaker-Menezes, D., Chiavarina, B., Zhou, J., et al. (2010). Autophagy in cancer associated fibroblasts promotes tumor cell survival: role of hypoxia, HIF1 induction and NFkappaB activation in the tumor stromal microenvironment. Cell Cycle 9, 3515-3533.

Martyniak, K., and Masternak, M. M. (2017). Changes in adipose tissue cellular composition during obesity and aging as a cause of metabolic dysregulation. Exp. Gerontol. 94, 59-63. doi: 10.1016/j.exger.2016.12.007

Milanovic, M., Fan, D. N. Y., Belenki, D., Dabritz, J. H. M., Zhao, Z., Yu, Y., et al. (2018). Senescence-associated reprogramming promotes cancer stemness. Nature 553, 96-100. doi: 10.1038/nature25167

Nelson, G., Kucheryavenko, O., Wordsworth, J., and von Zglinicki, T. (2018). The senescent bystander effect is caused by ROS-activated NFkappaB signalling. Mech. Ageing Dev. 170, 30-36. doi: 10.1016/j.mad.2017. 08.005

Nelson, G., Wordsworth, J., Wang, C., Jurk, D., Lawless, C., Martin-Ruiz, C., et al. (2012). A senescent cell bystander effect: senescence-induced senescence. Aging Cell 11, 345-349. doi: 10.1111/j.1474-9726.2012.00795.x

Nieman, K. M., Kenny, H. A., Penicka, C. V., Ladanyi, A., Buell-Gutbrod, R., Zillhardt, M. R., et al. (2011). Adipocytes promote ovarian cancer metastasis and provide energy for rapid tumor growth. Nat. Med. 17, 1498-1503. doi: $10.1038 / \mathrm{nm} .2492$

Ortiz-Montero, P., Londono-Vallejo, A., and Vernot, J. P. (2017). Senescenceassociated IL-6 and IL-8 cytokines induce a self- and cross-reinforced senescence/inflammatory milieu strengthening tumorigenic capabilities in the MCF-7 breast cancer cell line. Cell Commun. Signal. 15:17. doi: 10.1186/s12964017-0172-3

Parrinello, S., Coppe, J. P., Krtolica, A., and Campisi, J. (2005). Stromal-epithelial interactions in aging and cancer: senescent fibroblasts alter epithelial cell differentiation. J. Cell Sci. 118(Pt 3), 485-496.
Petruzzelli, M., Schweiger, M., Schreiber, R., Campos-Olivas, R., Tsoli, M., Allen, J., et al. (2014). A switch from white to brown fat increases energy expenditure in cancer-associated cachexia. Cell Metab. 20, 433-447. doi: 10.1016/j.cmet.2014. 06.011

Rodier, F., and Campisi, J. (2011). Four faces of cellular senescence. J. Cell Biol. 192, 547-556. doi: 10.1083/jcb.201009094

Rowan, B. G., Gimble, J. M., Sheng, M., Anbalagan, M., Jones, R. K., Frazier, T. P., et al. (2014). Human adipose tissue-derived stromal/stem cells promote migration and early metastasis of triple negative breast cancer xenografts. PLoS One 9:e89595. doi: 10.1371/journal.pone.008 9595

Ruhland, M. K., Loza, A. J., Capietto, A. H., Luo, X., Knolhoff, B. L., Flanagan, K. C., et al. (2016). Stromal senescence establishes an immunosuppressive microenvironment that drives tumorigenesis. Nat. Commun. 7:11762. doi: 10 . 1038/ncomms 11762

Serrano, M., Lin, A. W., McCurrach, M. E., Beach, D., and Lowe, S. W. (1997). Oncogenic RAS provokes premature cell senescence associated with accumulation of p53 and p16INK4a. Cell 88, 593-602.

Sieben, C. J., Sturmlechner, I., van de Sluis, B., and van Deursen, J. M. (2018). Two-step senescence-focused cancer therapies. Trends Cell Biol. 28, 723-737. doi: 10.1016/j.tcb.2018.04.006

Song, Y. H., Warncke, C., Choi, S. J., Choi, S., Chiou, A. E., Ling, L., et al. (2017). Breast cancer-derived extracellular vesicles stimulate myofibroblast differentiation and pro-angiogenic behavior of adipose stem cells. Matrix Biol. 60-61, 190-205. doi: 10.1016/j.matbio.2016. 11.008

Tchkonia, T., Morbeck, D. E., Von Zglinicki, T., Van Deursen, J., Lustgarten, J., Scrable, H., et al. (2010). Fat tissue, aging, and cellular senescence. Aging Cell 9, 667-684. doi: 10.1111/j.1474-9726.2010.00608.x

Tsuyada, A., Chow, A., Wu, J., Somlo, G., Chu, P., Loera, S., et al. (2012). CCL2 mediates cross-talk between cancer cells and stromal fibroblasts that regulates breast cancer stem cells. Cancer Res. 72, 2768-2779. doi: 10.1158/0008-5472. CAN-11-3567

Vazquez Rodriguez, G., Abrahamsson, A., Jensen, L. D. E., and Dabrosin, C. (2018). Adipocytes promote early steps of breast cancer cell dissemination via interleukin-8. Front. Immunol. 9:1767. doi: 10.3389/fimmu.2018. 01767

Wang, S., Wang, N., Zheng, Y., Zhang, J., Zhang, F., and Wang, Z. (2017). Caveolin-1: an oxidative stress-related target for cancer prevention. Oxid. Med. Cell. Longev. 2017:7454031. doi: 10.1155/2017/745 4031

Witkiewicz, A. K., Dasgupta, A., Sotgia, F., Mercier, I., Pestell, R. G., Sabel, M., et al. (2009). An absence of stromal caveolin-1 expression predicts early tumor recurrence and poor clinical outcome in human breast cancers. Am. J. Pathol. 174, 2023-2034. doi: 10.2353/ajpath.2009.080873

Wu, Q., Li, B., Li, Z., Li, J., Sun, S., and Sun, S. (2019a). Cancer-associated adipocytes: key players in breast cancer progression. J. Hematol. Oncol. 12:95.

Wu, Q., Li, J., Li, Z., Sun, S., Zhu, S., Wang, L., et al. (2019b). Exosomes from the tumour-adipocyte interplay stimulate beige/brown differentiation and reprogram metabolism in stromal adipocytes to promote tumour progression. J. Exp. Clin. Cancer Res. 38:223. doi: 10.1186/s13046-0191210-3

Wu, Q., Sun, S., Li, Z., Yang, Q., Li, B., Zhu, S., et al. (2019c). Breast cancer-released exosomes trigger cancer-associated cachexia to promote tumor progression. Adipocyte 8, 31-45. doi: 10.1080/21623945.2018.155 1688

Wu, Q., Sun, S., Li, Z., Yang, Q., Li, B., Zhu, S., et al. (2018). Tumouroriginated exosomal miR-155 triggers cancer-associated cachexia to promote tumour progression. Mol. Cancer 17:155. doi: 10.1186/s12943-0180899-5

Yang, G., Rosen, D. G., Zhang, Z., Bast, R. C. Jr., Mills, G. B., Colacino, J. A., et al. (2006). The chemokine growth-regulated oncogene 1 (Gro-1) links RAS signaling to the senescence of stromal fibroblasts and ovarian tumorigenesis. Proc. Natl. Acad. Sci. U.S.A. 103, 16472-16477.

Yang, H., Wang, H., Ren, J., Chen, Q., and Chen, Z. J. (2017). cGAS is essential for cellular senescence. Proc. Natl. Acad. Sci. U.S.A. 114, E4612-E4620. doi: 10.1073/pnas.1705499114 
Young, A. P., Schlisio, S., Minamishima, Y. A., Zhang, Q., Li, L., Grisanzio, C., et al. (2008). VHL loss actuates a HIF-independent senescence programme mediated by $\mathrm{Rb}$ and p400. Nat. Cell Biol. 10, 361-369. doi: 10.1038/ncb 1699

Young, A. R., Narita, M., Ferreira, M., Kirschner, K., Sadaie, M., Darot, J. F., et al. (2009). Autophagy mediates the mitotic senescence transition. Genes Dev. 23, 798-803. doi: 10.1101/gad.519709

Yu, D. M., Jung, S. H., An, H. T., Lee, S., Hong, J., Park, J. S., et al. (2017). Caveolin1 deficiency induces premature senescence with mitochondrial dysfunction. Aging Cell 16, 773-784. doi: 10.1111/acel.12606
Conflict of Interest: The authors declare that the research was conducted in the absence of any commercial or financial relationships that could be construed as a potential conflict of interest.

Copyright (c) $2020 \mathrm{Wu}, \mathrm{Li}$, Sun and Sun. This is an open-access article distributed under the terms of the Creative Commons Attribution License (CC BY). The use, distribution or reproduction in other forums is permitted, provided the original author(s) and the copyright owner(s) are credited and that the original publication in this journal is cited, in accordance with accepted academic practice. No use, distribution or reproduction is permitted which does not comply with these terms. 